\title{
LISA verification binaries with updated distances from Gaia Data Release 2
}

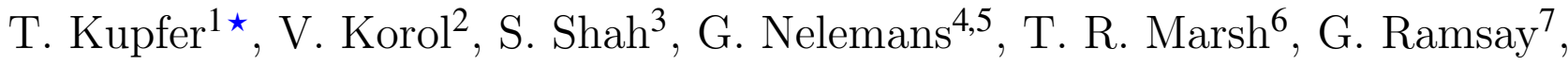 \\ P. J. Groot ${ }^{4}$, D. T. H Steeghs ${ }^{6}$, E. M. Rossi ${ }^{2}$, \\ ${ }^{1}$ Division of Physics, Mathematics and Astronomy, California Institute of Technology, Pasadena, CA 91125, USA \\ ${ }^{2}$ Leiden Observatory, Leiden University, PO Box 9513, 2300 RA, Leiden, the Netherlands \\ ${ }^{3}$ Albert-Einstein-Institut, Max-Planck-Institut fúr Gravitationsphysik, D-30167 Hannover, Germany \\ ${ }^{4}$ Department of Astrophysics/IMAPP, Radboud University Nijmegen, P.O.Box 9010, 6500 GL, Nijmegen, The Netherlands \\ ${ }^{5}$ Institute of Astronomy, KU Leuven, Celestijnenlaan 200D, B-3001 Leuven, Belgium \\ ${ }^{6}$ Department of Physics, University of Warwick, Coventry CV4 7AL, UK \\ ${ }^{7}$ Armagh Observatory and Planetarium, College Hill, Armagh, BT61 9DG,UK
}

Accepted XXX. Received YYY; in original form ZZZ

\begin{abstract}
Ultracompact binaries with orbital periods less than a few hours will dominate the gravitational wave signal in the $\mathrm{mHz}$ regime. Until recently, 10 systems were expected have a predicted gravitational wave signal strong enough to be detectable by the Laser Interferometer Space Antenna ( $L I S A)$, the so-called 'verification binaries'. System parameters, including distances, are needed to provide an accurate prediction of the expected gravitational wave strength to be measured by $L I S A$. Using parallaxes from Gaia Data Release 2 we calculate signal-to-noise ratios (SNR) for $\approx 50$ verification binary candidates. We find that 11 binaries reach a $\mathrm{SNR} \geq 20$, two further binaries reaching a $\mathrm{SNR} \geq 5$ and three more systems are expected to have a $\mathrm{SNR} \approx 5$ after four years integration with $L I S A$. For these 16 systems we present predictions of the gravitational wave amplitude $(\mathcal{A})$ and parameter uncertainties from Fisher information matrix on the amplitude $(\mathcal{A})$ and inclination $(\iota)$.
\end{abstract}

Key words: binaries: close - stars: distances - stars: individual: white dwarfs AM CVns

\section{INTRODUCTION}

The Laser Interferometer Space Antenna, (LISA) will be the first gravitational wave observatory in space (Amaro-Seoane et al. 2017). Operating in the low frequency part of the gravitational wave $(\mathrm{GW})$ spectrum $\left(10^{-4}-1 \mathrm{~Hz}\right)$, LISA has been selected as ESA's third large mission of the Cosmic Vision Program ${ }^{1}$. Amongst many other astrophysical sources, LISA will allow us to observe millions of ultracompact (Galactic) binaries (UCBs) with orbital periods $\left(\mathrm{P}_{\text {orb }}\right)$ shorter than a few hours (Amaro-Seoane et al. 2017) from which we will be able to individually resolve several thousands (e.g. Nelemans et al. 2004; Ruiter et al. 2009; Ruiter et al. 2010; Yu \& Jeffery 2010; Liu et al. 2010; Shah et al. 2012; Nissanke et al. 2012; Nelemans 2013; Littenberg et al. 2013; Kremer et al. 2017; Breivik et al. 2018). As indicated by their tight orbits, these systems are composed of degenerate stellar remnants, such as white dwarfs, neutron stars or stellar-mass black

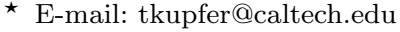

1 launch planned between 2030-2034
}

holes. Up to now several such sources have been detected in the electromagnetic (EM) bands. These include detached (Brown et al. 2016b) and semi-detached double white dwarfs (the latter called AM CVn type binaries; Solheim 2010), and semi-detached white dwarf-neutron star binaries (so-called ultracompact X-ray binaries; Nelemans \& Jonker 2010) and double neutron stars (Lyne et al. 2004).

A subset of the known UCBs have orbital periods that lie in the LISA band and these will be individually detected due to their strong GW signals. These LISAguaranteed sources are termed 'verification binaries' with some being expected to be detected on a timescale of weeks or a few months (Ströer \& Vecchio 2006). Therefore, they are crucial in facilitating the functional tests of the instrument and maximising the scientific output of LISA. So far we know of ten such systems, most of them being semi-detached AMCVn type: HM Cnc, V407 Vul, ES Cet, AM CVn, SDSS J190817.07+394036.4 (SDSS J1908), HP Lib, CR Boo, and V803 Cen (Strohmayer 2004; Ramsay et al. 2005; Espaillat et al. 2005; Roelofs et al. 2006, 2007c; Kupfer et al. 2015; Green et al. 
2018a). The remaining two are detached binary white dwarf systems: SDSS J065133.34+284423.4 (SDSS J0651) and SDSS J093506.92+441107.0 (SDSS J0935) (Brown et al. 2011; Kilic et al. 2014).

Predicting the gravitational wave strain depends on the masses of the binary components, which, together define the chirp mass (defined in $\S 3.3$ ), the orbital inclinations of the systems and their distance. Masses can be obtained, within limits, from optical spectroscopy and photometry, combined with the Roche-lobe geometry. In favourable situations, such as eclipsing systems, the orbital inclination can be determined from time-resolved spectroscopy and photometry (e.g. Brown et al. 2011), but it is generally rather poorly constrained. So far, distances remained the largest uncertainty. Only five of the known 52 semi-detached AM CVn type systems have HST-based parallaxes (Roelofs et al. 2007c): AM CVn, HP Lib, CR Boo, V803 Cen and GP Com. Groundbased parallaxes were derived for AM CVn (C.Dahn, as quoted by Nelemans et al. 2004), GP Com (Thorstensen 2003) and V396 Hya (Thorstensen et al. 2008). The remaining systems have distance estimates based on the comparison of model fluxes with the observations. These are considered highly uncertain as they require good knowledge of system parameters such as mass ratios, donor properties and accretion rates. Of the detached verification binary candidates, only one (WD 1242-105; Debes et al. 2015) has a parallax measurement. The remaining systems have indirect distance estimates based on the comparison of measured temperatures and surface gravities with stellar models (e.g. Althaus et al. 2013; Istrate et al. 2014; Brown et al. 2016c).

In April 2018, the Gaia collaboration released sky positions, parallaxes, and proper motions for more than 1.3 billion sources, with a limiting magnitude of $G=21 \mathrm{mag}$ (Gaia Collaboration et al. 2016, 2018). Here we present new results on the predicted gravitational wave signal detectable by $L I S A$ for known Galactic binaries using distances from Gaia Data Release 2 (DR2) and the current LISA baseline configuration. We calculate updated signal-to-noise (SNR) ratios. For the loudest known verification binaries with $\mathrm{SNR} \gtrsim 5$, we extract their GW parameter uncertainties using Fisher information matrix. For our analysis we define systems as verification binaries if the binary 1 ) is detected in the electromagnetic bands and 2) its SNR is $\geq 5$ after 4 years of integration which is the nominal mission time for LISA.

\section{THE SAMPLE OF VERIFICATION BINARIES}

Observationally, the known sample of 10 verification binaries is strongly biased and incomplete. This sample includes AM CVn, CR Boo, V803 Cen and ES Cet, which were all found as 'outliers' in surveys for blue, high-Galactic latitude stars. The selection effects are difficult to accurately quantify and therefore not easy to model. HM Cnc and V407 Vul are the most compact known systems and were discovered during the course of the ROSAT All-Sky Survey showing an on/off X-ray profile modulated on a period of 321 and 569 sec respectively (Israel et al. 1999; Motch et al. 1996). Their ultracompact nature was later confirmed with optical observations (Ramsay et al. 2002; Israel et al. 2002; Ramsay et al. 2000; Roelofs et al. 2010). SDSSJ1908 was found as a short- period variable in the original Kepler field, where it was targeted as a potential subdwarf B-star pulsator (Fontaine et al. 2011; Kupfer et al. 2015).

Extremely low mass (ELM) white dwarf binaries such as SDSS J0651 and SDSS J0935 were discovered as part of a colour selected sample of B-type hypervelocity candidates from the Sloan Digital sky survey (SDSS; Brown et al. 2016b and references therein). ELM white dwarfs can be separated efficiently from the bulk of white dwarfs with a colour selection.

Studies of UCBs have been conducted almost exclusively at high Galactic latitudes and in the Northern hemisphere. It is therefore likely that more 'AM CVn'-like systems are awaiting discovery in the Southern hemisphere and at low Galactic latitudes. Binary population studies predict that LISA will detect several thousand detached and semidetached double white dwarfs as well as a few tens of neutron star or black hole binaries with a population strongly peaking towards the Galactic Plane/Bulge (e.g. Nelemans et al. 2004). These studies suggest that about an equal fraction of semi-detached and detached systems are expected but the models over predict the number AM CVns observed in surveys like SDSS by at least a factor 10 (Roelofs et al. 2007b; Carter et al. 2013), so the detached systems may well dominate. Most of the detached systems are predicted to consist of a carbon/oxygen + helium white dwarf binary system (Nelemans et al. 2001b; Nelemans 2013; Ruiter et al. 2010; Liu et al. 2010; Yu \& Jeffery 2010).

Although the currently known sample is still limited, upcoming and ongoing large scale high-cadence variability surveys which also cover low Galactic latitudes such as OmegaWhite (Macfarlane et al. 2015), ZTF (Bellm 2014), BlackGEM (Bloemen et al. 2015), GOTO (Steeghs 2017), Gaia and LSST (see Korol et al. 2017 for both) have the potential to discover an unbiased sample of LISA verification binaries. Indeed Korol et al. (2017) show that Gaia, LSST and LISA have the potential to detect hundreds up to a few thousand new ultracompact double white dwarfs.

\section{METHODS}

\subsection{Mass assumption for systems without constraints}

Mass estimation for AM CVn type systems are difficult because only the accretion disc and in some cases the accretor is visible in the spectra. So far the only AMCVn systems with direct measurements of the donor and the accretor mass are eclipsing systems. Copperwheat et al. (2010) found precise masses for SDSS J092638.71+362402.4 and more recently Green et al. (2018b) derived precise masses for the first fully eclipsing AM CVn type system: Gaia 14aae. Both systems show a high accretor mass of 0.85 and $0.87 \mathrm{M}_{\odot}$ respectively. Additionally, both systems have donor stars which are inconsistent with a zero-temperature fully degenerate star. In both cases the donor is larger and more massive compared to what it is expected for a fully degenerate donor.

A large number of AM CVn systems have indirect constraints on the mass ratio $(q)$ from the empirical relation of the superhump excess (Knigge 2006).

$q=(0.114 \pm 0.005)+(3.97 \pm 0.41) \times(\epsilon-0.025)$ 
Table 1. Physical properties of the known verification binaries. Masses and inclination angles in brackets are assumed and based on evolutionary stage and mass ratio estimations

\begin{tabular}{|c|c|c|c|c|c|c|c|}
\hline Source & $\begin{array}{r}l_{\mathrm{Gal}} \\
(\mathrm{deg})\end{array}$ & $\begin{array}{r}b_{\mathrm{Gal}} \\
(\mathrm{deg})\end{array}$ & $\begin{array}{r}\text { Orbital period } \\
(\mathrm{sec})\end{array}$ & $\begin{array}{l}m_{1} \\
\left(\mathrm{M}_{\odot}\right)\end{array}$ & $\begin{array}{l}m_{2} \\
\left(\mathrm{M}_{\odot}\right)\end{array}$ & $\stackrel{\iota}{(\operatorname{deg})}$ & Refs. \\
\hline \multicolumn{8}{|l|}{ AM CVn type } \\
\hline HM Cnc & 206.9246 & 23.3952 & 321.529 & 0.55 & 0.27 & $\approx 38$ & 1,2 \\
\hline V407 Vul & 57.7281 & 6.4006 & 569.395 & {$[0.8 \pm 0.1]$} & {$[0.177 \pm 0.071]$} & {$[60]$} & 3 \\
\hline ES Cet & 168.9684 & -65.8632 & 620.21 & {$[0.8 \pm 0.1]$} & {$[0.161 \pm 0.064]$} & {$[60]$} & 4 \\
\hline SDSS J135154.46-064309.0 & 328.5021 & 53.1240 & 943.84 & {$[0.8 \pm 0.1]$} & {$[0.100 \pm 0.040]$} & {$[60]$} & 5 \\
\hline $\mathrm{AM} C V n$ & 140.2343 & 78.9382 & 1028.73 & $0.68 \pm 0.06$ & $0.125 \pm 0.012$ & $43 \pm 2$ & 6,7 \\
\hline SDSS J190817.07+394036.4 & 70.6664 & 13.9349 & 1085.7 & {$[0.8 \pm 0.1]$} & {$[0.085 \pm 0.034]$} & $10-20$ & 8,9 \\
\hline HP Lib & 352.0561 & 32.5467 & 1102.70 & $0.49-0.80$ & $0.048-0.088$ & $26-34$ & 10,11 \\
\hline PTF1 J191905.19+481506.2 & 79.5945 & 15.5977 & 1347.35 & {$[0.8 \pm 0.1]$} & {$[0.066 \pm 0.026]$} & {$[60]$} & 12 \\
\hline CXOGBS J175107.6-294037 & 359.9849 & -1.4108 & 1375.0 & {$[0.8 \pm 0.1]$} & {$[0.064 \pm 0.026]$} & {$[60]$} & 13 \\
\hline CR Boo & 340.9671 & 66.4884 & 1471.3 & $0.67-1.10$ & $0.044-0.088$ & 30 & 11,14 \\
\hline V803 Cen & 309.3671 & 20.7262 & 1596.4 & $0.78-1.17$ & $0.059-0.109$ & $12-15$ & 11,15 \\
\hline \multicolumn{8}{|l|}{ Detached white dwarfs } \\
\hline SDSS J065133.34+284423.4 & 186.9277 & 12.6886 & 765.5 & $0.247 \pm 0.015$ & $0.49 \pm 0.02$ & $86.9_{-10}^{+1.6}$ & 16,17 \\
\hline SDSS J093506.92+441107.0 & 176.0796 & 47.3776 & 1188.0 & $0.312 \pm 0.019$ & $0.75 \pm 0.24$ & {$[60]$} & 18,19 \\
\hline SDSS J163030.58+423305.7 & 67.0760 & 43.3604 & 2389.8 & $0.298 \pm 0.019$ & $0.76 \pm 0.24$ & {$[60]$} & 18,20 \\
\hline SDSS J092345.59+302805.0 & 195.8199 & 44.7754 & 3883.7 & $0.275 \pm 0.015$ & $0.76 \pm 0.23$ & {$[60]$} & 18,21 \\
\hline \multicolumn{8}{|l|}{ Hot subdwarf binaries } \\
\hline CD $-30^{\circ} 11223$ & 322.4875 & 28.9379 & 4231.8 & $0.54 \pm 0.02$ & $0.79 \pm 0.01$ & $82.9 \pm 0.4$ & 22 \\
\hline
\end{tabular}

[1]Strohmayer (2005), [2]Roelofs et al. (2010), [3]Ramsay et al. (2002), [4]Espaillat et al. (2005), [5]Green et al. (2018a), [6]Skillman et al. (1999), [7]Roelofs et al. (2006), [8]Fontaine et al. (2011), [9]Kupfer et al. (2015), [10]Patterson et al. (2002), [11]Roelofs et al. (2007c), [12]Levitan et al. (2014),[13]Wevers et al. (2016), [14]Provencal et al. (1997), [15]Roelofs et al. (2007a), [16]Brown et al. (2011), [17]Hermes et al. (2012), [18]Brown et al. (2016c), [19]Kilic et al. (2014), [20]Kilic et al. (2011), [21](Brown et al. 2010), [22]Geier et al. (2013)

where $\epsilon=\frac{P_{\mathrm{sh}}-P_{\text {orb }}}{P_{\mathrm{orb}}}$ is the superhump excess. This is an empirical relation which gives similar results to that of Patterson et al. (2005) but with the inclusion of uncertainties on the fit parameters. The relation was derived for hydrogendominated cataclysmic variables but has not yet been well tested for AM CVn type systems. Green et al. (2018a) applied the equation to 11 AMCVn systems with a measured superhump excess to derive the mass and radius for the donor under the assumption of an $0.7 \pm 0.1 \mathrm{M}_{\odot}$ accretor. None of the tested systems are consistent with a fully degenerate donor but they are on average about 2.5 times the mass compared to a zero-temperature fully degenerate donor.

Based on this result and the measurements from the eclipsing systems we assume for systems without constraints on the component masses an accretor mass of $0.8 \pm 0.1 \mathrm{M}_{\odot}$ and a donor mass 2.5 times the mass for a zero-temperature donor star. For the donor star we allow an error range of 1.5 - 3.5 times the minimum mass. System properties for each system are given in Table 1.

\subsection{Distance determination from Gaia DR2 parallaxes}

Gaia DR2 provides parallaxes, not distances. In this Section we explain the procedure we adopt to convert parallaxes into distances. To estimate distances from the measured parallaxes a probability-based inference approach is required (e.g. Bailer-Jones 2015; Igoshev et al. 2016; Astraatmadja \& Bailer-Jones 2016; Bailer-Jones et al. 2018;
Luri et al. 2018). Essentially, because any measured parallax $(\varpi)$ follows a probability distribution, we can infer the distance in a probabilistic sense, if we make an assumption about the true distribution of observed sources in space (i.e. the prior distribution). Using Bayes' theorem the posterior probability density of the possible values for the distance can be expressed as

$$
\begin{array}{r}
P\left(d \mid \varpi, \sigma_{\varpi}\right)=\frac{1}{Z} P\left(\varpi \mid d, \sigma_{\varpi}\right) P(d) ; \\
Z=\int_{0}^{\infty} P\left(\varpi \mid r, \sigma_{\varpi}\right) P(r) d r,
\end{array}
$$

where $Z$ is the normalization constant, $P\left(\varpi \mid d, \sigma_{\varpi}\right)$ is the likelihood function and $P(d)$ is the prior. The likelihood expresses the probability to measure the parallax $\varpi$ for the source at the distance $d$ with an uncertainty of the measurement $\sigma_{\varpi}$. For Gaia measurements we can assume a Gaussian noise model (Lindegren et al. 2018) and write the likelihood as

$P\left(\varpi \mid d, \sigma_{\varpi}\right)=\frac{1}{\sqrt{2 \pi} \sigma_{\varpi}} \exp \left[-\frac{1}{2 \sigma_{\varpi}^{2}}\left(\varpi-\frac{1}{d}\right)^{2}\right]$.

The prior $P(d)$ contains our assumption about the distance distribution of the sources. For measurements with fractional parallax errors $\sigma_{\varpi} / \varpi$ less than about $0.1-0.2$, the distance estimates are mainly independent of the choice of prior. However, for larger fractional errors the quality of the distance estimates heavily depends on how well the prior reflects the true distribution of distances for the population of sources (e.g. Bailer-Jones 2015; Astraatmadja \& Bailer- 
Table 2. Measured EM properties (parallax, distance) and derived GW parameters (f, $\mathcal{A}$, signal-to-noise ratio SNR) of the known verification binaries. The distance for HM Cnc is assumed. The strain amplitude $(\mathcal{A})$ is given in units of $10^{-23}$. The $\mathrm{SNR}$ is calculated for four years integration with LISA.

\begin{tabular}{lrrrrrrr}
\hline Source & $\begin{array}{r}f \\
(\mathrm{mHz})\end{array}$ & $\begin{array}{r}\varpi \\
(\mathrm{mas})\end{array}$ & $\begin{array}{r}\sigma_{\varpi} \\
(\mathrm{mas})\end{array}$ & $\begin{array}{r}d \\
(\mathrm{pc})\end{array}$ & $\begin{array}{r}\sigma_{d} \\
(\mathrm{pc})\end{array}$ & $\mathcal{A}$ & SNR \\
\hline AM CVn type systems & & & & & & & \\
HM Cnc & 6.22 & - & - & {$[5000]$} & - & 6.4 & $211.1 \pm 3.18$ \\
V407 Vul & 3.51 & 0.095 & 0.327 & 1786 & 667 & $11.0 \pm 5.9$ & $169.7 \pm 2.17$ \\
ES Cet & 3.22 & 0.596 & 0.108 & 1584 & 291 & $10.7 \pm 4.6$ & $154.3 \pm 2.09$ \\
SDSS J135154.46-064309.0 & 2.12 & 0.596 & 0.313 & 1317 & 531 & $6.2 \pm 3.5$ & $21.8 \pm 0.24$ \\
AM CVn & 1.94 & 3.351 & 0.045 & 299 & 4 & $28.3 \pm 3.2$ & $101.2 \pm 0.96$ \\
SDSS J190817.07+394036.4 & 1.84 & 0.954 & 0.046 & 1044 & 51 & $6.1 \pm 2.4$ & $20.3 \pm 0.13$ \\
HP Lib & 1.81 & 3.622 & 0.052 & 276 & 4 & $17.5 \pm 3.9$ & $43.7 \pm 0.28$ \\
PTF1 J191905.19+481506.2 & 1.48 & 0.550 & 0.327 & 1338 & 555 & $3.2 \pm 1.8$ & $4.0 \pm 0.02$ \\
CXOGBS J175107.6-294037 & 1.45 & 1.016 & 0.146 & 971 & 156 & $4.2 \pm 1.8$ & $4.5 \pm 0.02$ \\
CR Boo & 1.36 & - & - & $337^{a}$ & -34 & 13.4 \\
V803 Cen & 1.25 & - & - & $347^{a}$ & -27 & $16.0 \pm 5.4$ & $21.9 \pm 0.13$ \\
detached white dwarfs & & & & & & & \\
SDSS J065133.34+284423.4 & 2.61 & 1.000 & 0.476 & 933 & 493 & $16.2 \pm 8.6$ & $90.1 \pm 1.13$ \\
SDSS J093506.92+441107.0 & 1.68 & - & - & $645^{b}$ & $41^{b}$ & $29.9 \pm 7.7$ & $44.9 \pm 0.31$ \\
SDSS J163030.58+423305.7 & 0.84 & 0.937 & 0.270 & 1019 & 357 & $11.5 \pm 4.9$ & $4.6 \pm 0.03$ \\
SDSS J092345.59+302805.0 & 0.51 & 3.340 & 0.173 & 299 & 10 & $26.4 \pm 6.5$ & $5.6 \pm 0.06$ \\
hot subdwarf binaries & & & & & & & \\
CD-3011223 & 0.47 & 2.963 & 0.080 & 337 & 9 & $41.5 \pm 1.8$ & $4.9 \pm 0.04$ \\
\hline
\end{tabular}

${ }^{a}$ Roelofs et al. (2007c), ${ }^{b}$ Brown et al. (2016a)

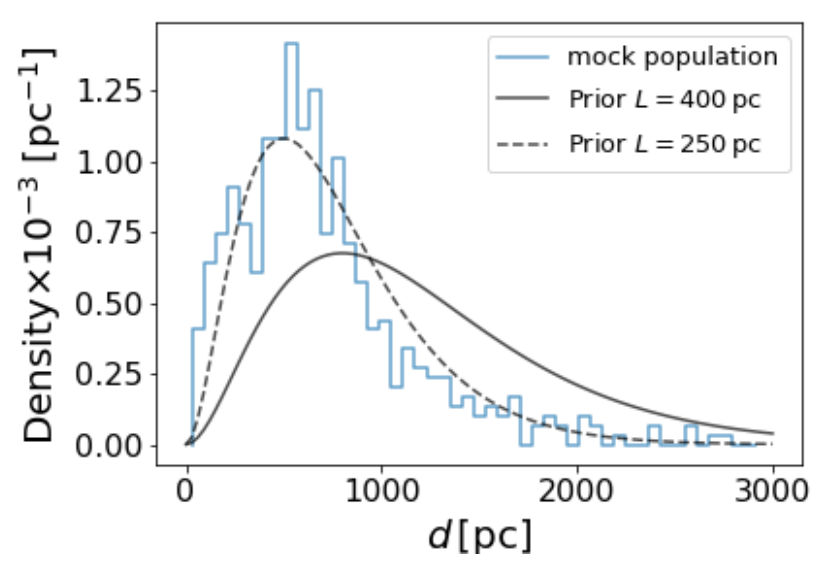

Figure 1. Distribution of synthetic detached double white dwarf binaries with distance from Korol et al. (2017) (blue line) and two exponentially decreasing volume density priors: $L=250 \mathrm{pc}$ (dashed black line) and $L=400 \mathrm{pc}$ (solid black line).

Jones 2016). For this work we adopt an exponentially decreasing volume density prior

$P(d)= \begin{cases}\frac{d^{2}}{2 L^{3}} \exp (-d / L) & \text { if } d>0, \\ 0 & \text { otherwise }\end{cases}$

where $L>0$ is the scale length. This prior performs well for a generic population, but fine-tuning is required to find the appropriate scale length $\mathrm{L}$ that describes $L I S A$ verification binaries (Astraatmadja \& Bailer-Jones 2016). We calibrate the value of $L$ using the mock catalog of detached double white dwarf binaries from Korol et al. (2017). The catalog was obtained using the binary population synthesis model of Nelemans et al. (2001a); Toonen et al. (2017) and designed to test the detectability of these binaries by Gaia, LSST and LISA. We select binaries in the catalog with Gaia $G$ magnitude $<21$ and where parallax fractional error ${ }^{2}>0.2$. A straightforward way of fine-tuning the value of $\mathrm{L}$ is to fit the distribution of synthetic binaries with the distance. Another way consists of finding the value of $L$ that minimizes the bias on our estimates due to a particular choice for $L$ itself (e.g. Marchetti et al. 2018). The latter implies the following calculations. For each binary we determine the mode of the posterior distribution $P\left(d \mid \varpi, \sigma_{\varpi}\right)$. The mode is an unbiased estimator and provides meaningful estimates when the posterior is highly asymmetric. To determine the mode of $P\left(d \mid \varpi, \sigma_{\varpi}\right)$ given our choice of the prior, we set the derivative of the posterior to be equal to zero and solve the equation (Bailer-Jones 2015):

$\frac{d^{3}}{L}-2 d^{2}+\frac{\varpi d}{\sigma_{\varpi}^{2}}-\frac{1}{\sigma_{\varpi}^{2}}=0$.

We repeat this calculation using a range of values for $L$ for each binary in the mock catalog. We define the best value of $L$ as the one that minimizes the difference between the mode, obtained by solving Eq. (5), and the true distance of the binary in the catalog. We obtain $L=400 \mathrm{pc}$. In Figure 1 we show the distribution of synthetic binaries with the distance (blue line) and two exponentially decreasing volume density priors: one with $L=250 \mathrm{pc}$ (dashed black line), that represents the best fit to the distribution of mock binaries, and another one with $L=400 \mathrm{pc}$ (solid black line), obtained

2 The errors on parallax for the mock population are estimated using PYGAIA python tool kit. 


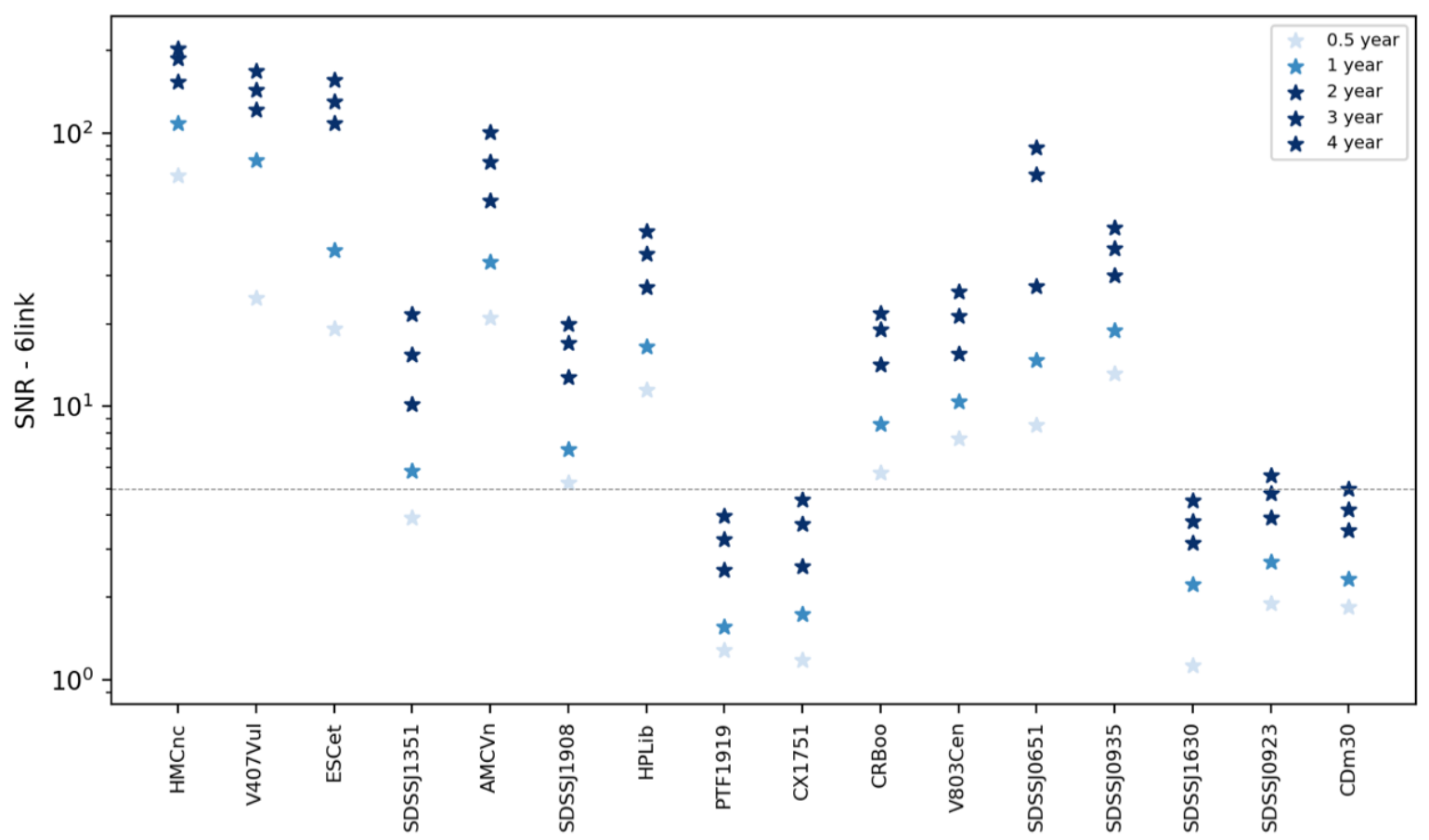

Figure 2. SNR evolution with time for the LISA verification binaries. The black dashed line corresponds to SNR=5.

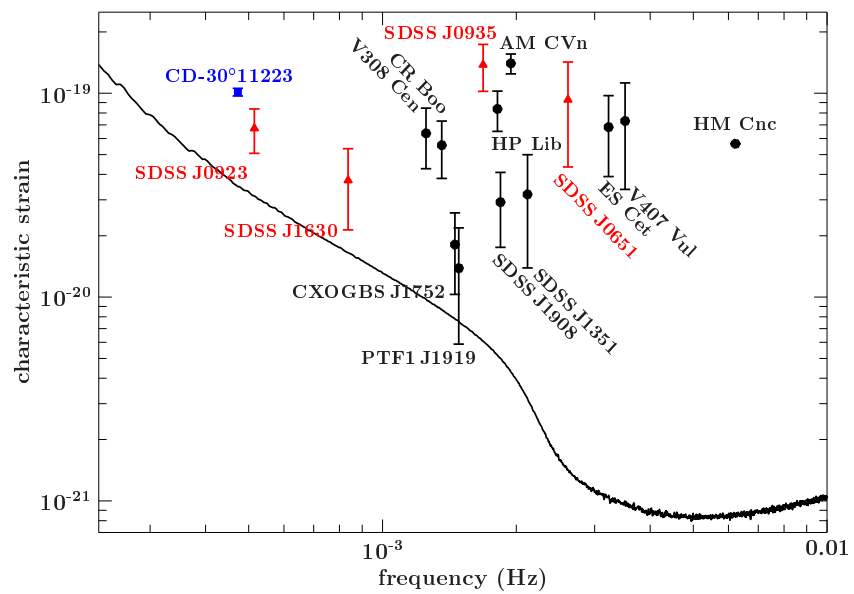

Figure 3. Sensitivity plot for LISA adopted assuming 4 years integration from Amaro-Seoane et al. (2017) showing the verification binaries which reach a $\mathrm{SNR} \geq 5$ or are on the border to a $\mathrm{SNR} \approx 5$ after 4 years integration. Black circles are AM CVn systems, red triangles correspond to detached white dwarfs and the blue square is the hot subdwarf binary. Note that the gravitational frequency shown here is twice the orbital frequency of the binaries. We assume a distance of $5 \mathrm{kpc}$ for HM Cnc.

by minimazing the bias. The figure shows that the curve with $L=400 \mathrm{pc}$ decreases slower and is more representative of binaries at large distances, where fractional errors on parallax are large. Thus, for this work we adopt the scale length of $400 \mathrm{pc}$ such that we avoid underestimating distances for the furthest binaries. Finally, following Bailer-Jones (2015) we associate the most probable value of $d$ with the mode of the posterior distribution, and we compute the errors as

$\sigma_{d}=\frac{d_{95}-d_{5}}{2 s}$,

where $d_{95}$ and $d_{5}$ are the boundaries of the $90 \%$ credible interval of the $P\left(d \mid \varpi, \sigma_{\varpi}\right)$ distribution that are calculated symmetrically about the median and $s=1.645$, which is the ratio of the $90 \%$ to $68.3 \%$ credible interval for a Gaussian distribution. HM Cnc, CR Boo, V803 Cen and SDSS J093506.92+441107.0 have no measured parallax from Gaia DR2. For HM Cnc we assumed $5 \mathrm{kpc}$ and discuss the uncertainty on the distance in detail in $\S 5$. For the other three systems the previously published distant estimates were used. The results are listed in Table 2 .

\subsection{Strain and SNR calculations}

To compute the expected characteristic strain we first calculate the dimensionless gravitational wave amplitude $(\mathcal{A})$ using equation 3 from Shah et al. (2012):

$\mathcal{A}=\frac{2(G \mathcal{M})^{5 / 3}}{c^{4} d}(\pi f)^{2 / 3}$

where $\mathcal{M}$ is the chirp mass, $\mathcal{M} \equiv\left(m_{1} m_{2}\right)^{3 / 5} /\left(m_{1}+m_{2}\right)^{1 / 5}, m_{1}$ and $m_{2}$ the masses of the two components, where we assume $m_{1}>m_{2}, d$ is the distance to the source as defined in $\S 3.2$ and $f$ the gravitational wave frequency with $f=2 / P_{\text {orb }}$. The characteristic strain $\left(h_{c}\right)$ for individual verification binaries was calculated following the approach described in Section 2 in Moore et al. (2015):

$h_{c}=\sqrt{N_{\text {cycle }}} \mathcal{A}$ 


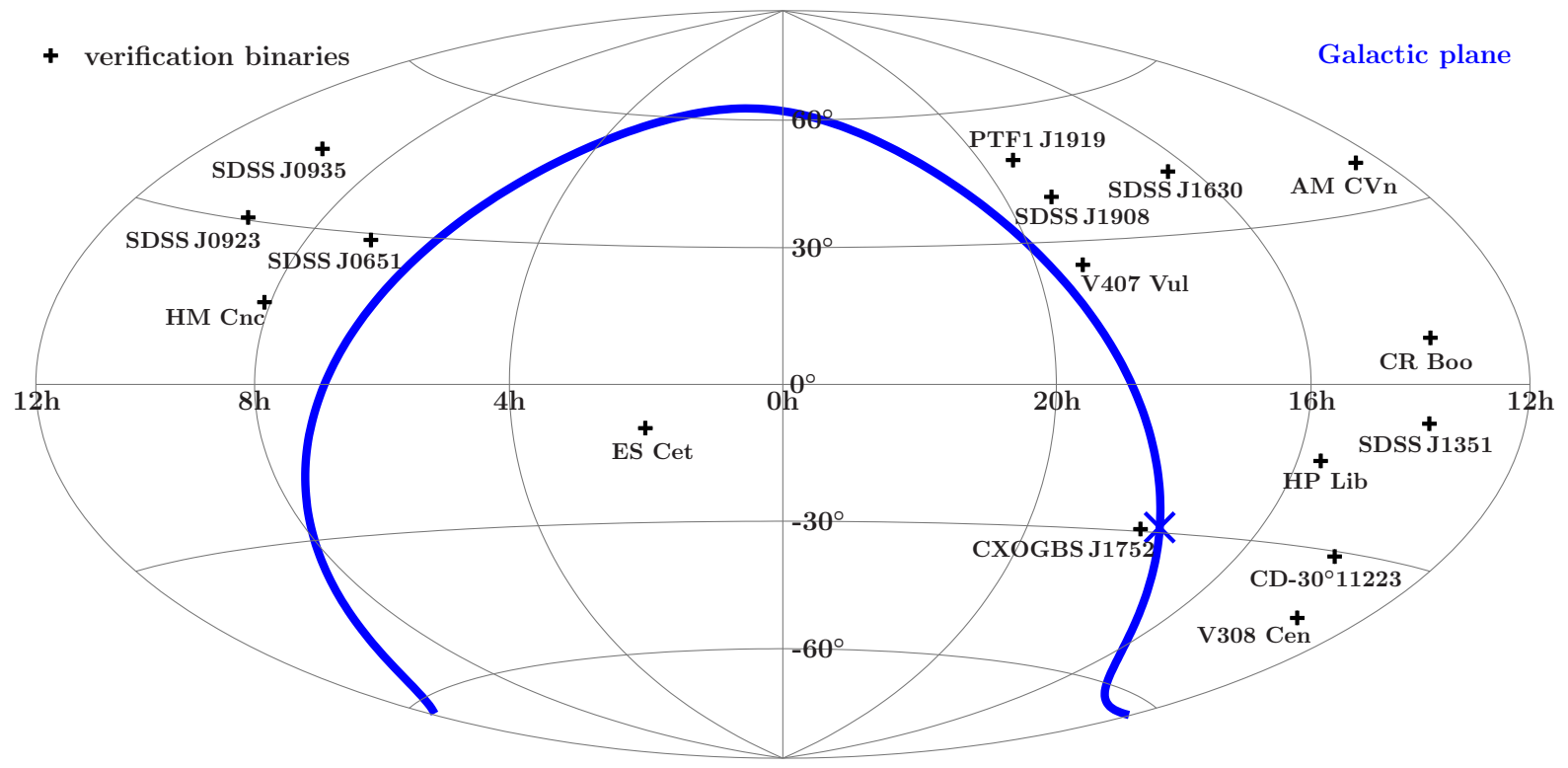

Figure 4. Sky position of the verification binaries. The sky positions show a clear bias towards the Northern hemisphere and to higher Galactic latitudes. The blue line indicates the Galactic Plane, with the Galactic Center located at the blue cross.

where $N_{\text {cycle }}=f T_{\text {obs }}$. For the calculations we assume that LISA will observe for four years. The masses and gravitational wave frequency for each system are given in Tables 1 and 2 .

Most of the LISA verification binaries can be characterized as monochromatic GW signals with a set of seven parameters, $\mathcal{A}, f$, polarization angle $(\psi)$, initial GW phase $\left(\phi_{0}\right)$, orbital inclination $(\iota)$, ecliptic latitude $(\sin \beta)$, and ecliptic longitude $(\lambda)$. An additional eighth parameter, the period derivative or chirp $(\dot{f})$ is used for HM Cnc, V407 Vul and SDSS J0651 which have measured orbital decay rates from their EM data, $\dot{P}_{\text {orb }}: 3.75 \times 10^{-11}{s s^{-1}}^{-1}$ Roelofs et al. 2010 ), $3.17 \times 10^{-12} s^{-1}$ (Ramsay et al. 2005), $9.8 \pm 2.8 \times$ $10^{-12}{s s^{-1}}^{(H e r m e s ~ e t ~ a l . ~ 2012) ~ r e s p e c t i v e l y . ~ T h e y ~ a r e ~ r e l a t e d ~}$ to the GW decay rate, $\dot{f}$ by $-\dot{P}_{\text {orb }} / P_{\text {orb }}^{2}$ used in simulating their GW signals. We compute Fisher matrices (e.g Cutler 1998) to extract the GW parameter uncertainties and correlations. The method and application of Fisher information matrix (FIM) for the LISA compact binaries together with their signal modeling and the noise from the detector and the Galactic foreground have been described in detail in Shah et al. (2012).

Here we use the current configuration for the LISA detector (Amaro-Seoane et al. 2017) with armlengths of $2.5 \times 10^{6} \mathrm{~km}$ and six laser links exchanged along the three arms of the detector, from which we can generate two sets of the optimal data streams from two channels yielding two independent time-series whose noises are uncorrelated maximizing the $\mathrm{SNR}^{3}$. Detailed discussions of the possible data streams using various sets of laser links can be found in e.g Vallisneri (2005). The unresolved foreground is obtained by using the recently updated catalog for detached double white dwarf binaries whose simulation and binary evolution is described in Toonen et al. (2017).

${ }^{3}$ We use the Time Delay Interferometry (TDI) A and E observables
We obtain the SNR from the GW signal over 15 instrumental noise realizations for the bright verification binaries using the nominal EM measurements to estimate the GW parameters in the GW signal model. For details we refer to $\S 3$ in Shah et al. (2012). Given the GW signal of the binary and a Gaussian noise we can use FIM to estimate the parameter uncertainties. The inverse of the FIM is the variance-covariance matrix whose diagonal elements are the GW uncertainties and the off-diagonal elements are the correlations between the two parameters. We do the GW analysis of the above mentioned verification binaries for LISA observations of four years. We note that the Fisher-based method is a quick way of computing parameter uncertainties and their correlations in which these uncertainties are estimated locally at the true parameter values and therefore by definition the method cannot be used to sample the entire posterior distribution of the parameters. Additionally Fisher-based results hold in the limit of strong signals with a Gaussian noise (see the Appendix in Shah \& Nelemans $2014)^{4}$.

\section{RESULTS}

We calculate the distance and expected SNR following the description outlined in $\S 3$ for $\approx 50$ semi-detached and detached candidate verification binaries with the strongest expected gravitational wave signals. Table 2 presents the predicted gravitational wave amplitude $(\mathcal{A})$ as well as the expected SNR after 4 years integration with LISA for all systems with $\mathrm{SNR} \geq 5$ and systems which are on the border to a $\mathrm{SNR} \approx 5$.

We find that 13 systems reach a $\mathrm{SNR} \geq 5$ after four
4 The code used to perform the simulation is available at https://doi.org/10.17617/1.68 
Table 3. GW parameter uncertainties for the bright verification binaries from Fisher Information Matrix after four years of LISA integration

\begin{tabular}{lrrrr}
\hline \hline Source & SNR & $\sigma_{\mathcal{A} / \mathcal{A}}$ & $\sigma_{\iota}\left[{ }^{\circ}\right]$ & $c_{\mathcal{A} \iota}$ \\
\hline HM Cnc & $211.1 \pm 3.18$ & $0.07 \pm 0.001$ & $5.82 \pm 0.08$ & $0.991 \pm 0.029$ \\
V407 Vul & $169.7 \pm 2.17$ & $0.028 \pm 0.000$ & $1.34 \pm 0.02$ & $0.907 \pm 0.023$ \\
ES Cet & $154.3 \pm 2.09$ & $0.032 \pm 0.000$ & $1.44 \pm 0.02$ & $0.911 \pm 0.024$ \\
SDSS J135154.46-064309.0 & $21.8 \pm 0.24$ & $0.218 \pm 0.002$ & $10.22 \pm 0.11$ & $0.911 \pm 0.020$ \\
AM CVn & $101.2 \pm 0.96$ & $0.113 \pm 0.001$ & $8.03 \pm 0.08$ & $0.985 \pm 0.018$ \\
SDSS J190817.07+394036.4 & $20.3 \pm 0.13$ & $5.622 \pm 0.036$ & $-a$ & $1.000 \pm 0.013$ \\
HP Lib & $43.7 \pm 0.28$ & $0.599 \pm 0.004$ & $63.82 \pm 0.41$ & $0.997 \pm 0.013$ \\
PTF1 J191905.19+481506.2 & $4.0 \pm 0.02$ & $1.218 \pm 0.008$ & $57.54 \pm 0.33$ & $0.909 \pm 0.011$ \\
CXOGBS J175107.6-294037 & $4.5 \pm 0.02$ & $1.057 \pm 0.005$ & $49.65 \pm 0.33$ & $0.909 \pm 0.009$ \\
CR Boo & $21.9 \pm 0.13$ & $1.173 \pm 0.007$ & $126.05 \pm 0.72$ & $0.997 \pm 0.011$ \\
V803 Cen & $26.2 \pm 0.17$ & $4.647 \pm 0.029$ & $-a$ & $1.000 \pm 0.013$ \\
SDSS J065133.34+284423.4 & $90.1 \pm 1.13$ & $0.022 \pm 0.000$ & $0.65 \pm 0.01$ & $0.159 \pm 0.004$ \\
SDSS J092345.59+302805.0 & $44.9 \pm 0.31$ & $0.106 \pm 0.001$ & $4.99 \pm 0.03$ & $0.909 \pm 0.013$ \\
SDSS J163030.58+423305.7 & $4.6 \pm 0.03$ & $1.064 \pm 0.008$ & $49.29 \pm 0.39$ & $0.909 \pm 0.014$ \\
SDSS J092345.59+302805.0 & $5.6 \pm 0.06$ & $0.834 \pm 0.009$ & $39.51 \pm 0.44$ & $0.908 \pm 0.020$ \\
CD-30 11223 & $4.9 \pm 0.04$ & $0.425 \pm 0.004$ & $12.52 \pm 0.13$ & $0.359 \pm 0.007$ \\
\hline
\end{tabular}

$a$ The FIM uncertainty exceeds the physically allowed range by $\iota$ and thus cannot be determined from GW data analysis Shah \& Nelemans (2014)

years observing with LISA and therefore are confirmed verification binaries based on the definition adopted in $\S 1$. The population consists of 9 AMCVn binaries: HM Cnc, V407 Vul, ES Cet, SDSS J1351, AM CVn, SDSS J1908, HP Lib, CR Boo and V803 Cen, 3 double white dwarfs: SDSS J0651, SDSS J0935 and SDSS J0923 as well as CD$30^{\circ} 11223$ the first verification binary consisting of a hot subdwarf star with a massive white dwarf companion. Additionally, we find three more systems (PTF1 J1919, CXOGBS J1751 and SDSS J1630) with a SNR of $\approx 5$ or just below 5 , making them good candidates for being verification binaries.

Figure 2 shows the evolution of the SNR after 0.5, 1, 2,3 and 4 years. The loudest source is HM Cnc which reaches a $\mathrm{SNR}=211$ after four years of integration and already $\mathrm{SNR}=69$ after 0.5 years, but whose distance is, even after Gaia DR2, still poorly constrained. PTF1 J1919, CXOGBS J1751, SDSS J1630, SDSS J0923 and CD-30 11223 need four years of integration to reach a SNR $\approx 5$. Figure 3 shows the LISA sensitivity curve and the characteristic strain of the verification binaries after four years observing with LISA.

Table 3 shows the parameter uncertainties extracted from the FIM. Of the seven GW parameters characterizing a binary, the astrophysically interesting ones are the amplitude $(\mathcal{A})$ and the inclination $(\iota)$. Shown are the relative $1-\sigma$ error in $\mathcal{A}$, absolute $1-\sigma$ error in $\iota$ and the normalized correlation between the two parameters $c_{\mathcal{A}} \iota$. The SNR influences the parameter uncertainties. Then the correlation $c_{\mathcal{A}} \iota$ has a strong influence on their uncertainties (Shah et al. 2012). As a result the systems with lower inclinations (or face-on orientations) with $\iota=\left[0^{\circ}-45^{\circ}\right]$ have a strong correlation due to the GW signals being indistinguishable by making changes in $\mathcal{A}$ or $\iota$. This explains the large errors in $\mathcal{A}$ and undetermined $\iota$ (since the GW uncertainties are greater than the physical values $\iota$ can take: $0^{\circ}-360^{\circ}$ ) for systems such as SDSS J190817.07+394036.4, CR Boo and V803 Cen despite that their SNRs are greater than 20. Whereas CD-30¹1223 has a better constrained inclination even though its SNR is lower at $\approx 5$.

\section{DISCUSSION}

The only remaining system without a distance measurement is HM Cnc: distance is therefore the largest uncertainty when predicting its $\mathcal{A}$ and SNR for LISA. Given its known properties we argue that $10 \mathrm{kpc}$ is the most conservative estimation for the distance. Although $\mathcal{A}$ and SNR remain uncertain, we find that even at a distance of $10 \mathrm{kpc}$, HM Cnc will have a $\mathrm{SNR} \approx 100$ after four years observing with $L I S A$. Hence it remains a bright verification binary even if the distance is significantly greater than the assumed $5 \mathrm{kpc}$.

Although there is a Gaia parallax measurement for V407 Vul (0.095 \pm 0.327$)$, the optical counterpart is dominated by a component that matches a G-type star, with a blue variable component in phase with the binary making up only $10-40 \%$ of the flux (Steeghs et al. 2006). The probability that this is an unrelated chance alignment of a foreground object is small, but the specific association of this G-star component with the ultra-compact binary is unclear. Given that this star dominates the Gaia passband, we assume here that the parallax measurement for V407 Vul is essentially that of the G-star component, and adopt this also for the ultra-compact binary component given the close on-sky alignment.

With the current LISA configuration and four years of observation, $>27,000^{5}$ binaries are expected to be individually detected by LISA. However, only a small fraction will be bright enough to be detectable in the optical.

5 Estimate from the foreground simulation using Toonen et al. (2017) catalogue 
Nelemans et al. (2004) predict that 143 short period semidetached $L I S A$ verification systems (3 in the direct-impact phase) with $\mathrm{P}_{\text {orb }}<1500 \mathrm{~s}$ and brighter than 20 mag should be detectable in the optical wavebands. More recent work by Korol et al. (2017) predict several tens, up to one hundred, detached double white dwarfs will be detectable in the optical bands by Gaia and LSST as eclipsing sources, those with high SNR from their gravitational waves and brighter than 24 mag. The eclipsing systems only represent a small fraction of the full sample and we expect that there are about 100 detached double white dwarfs with orbital periods below 10 min and brighter than 24 mag and therefore potentially detectable with LSST.

Since verification binaries are a Galactic population their surface density is expected to strongly peak near the Galactic Plane. Most of the known systems are located in the Northern hemisphere and only a few systems were found at low Galactic latitudes. This shows that the current sample is likely very incomplete and biased. Figure 4 shows the sky position of the 16 systems. Upcoming and ongoing large scale optical surveys such as OmegaWhite (Macfarlane et al. 2015), ZTF (Bellm 2014), BlackGEM (Bloemen et al. 2015), GOTO (Steeghs 2017), Gaia and LSST (see Korol et al. 2017 for both) are expected to discover a more unbiased sample across both hemispheres and at low Galactic latitudes before LISA gets launched.

\section{SUMMARY AND CONCLUSIONS}

In this work we derived distances from Gaia DR2 parallaxes for $\approx 50$ verification binary candidates. Using these distances, we calculated the expected SNR after four years integration with LISA with a configuration of 6 laser links and $2.5 \mathrm{Gm}$ arm lengths. Given the definition of a verification binary as $\mathrm{SNR} \geq 5$ after four years integration, we find a total of 13 verification binaries. Eleven systems reach a $S N R \geq 20$ and two additional systems reach a $\mathrm{SNR} \geq 5$ after four years. Additionally we find three more systems which are expected to have a $\mathrm{SNR} \approx 5$ after four years integration with $L I S A$ and are good candidates for being verification binaries. Our study confirmed the first hot subdwarf binary as a LISA verification binary.

So far, distances have been the most uncertain parameter when predicting the gravitational wave strengths of the bright verification binaries. This is in particular true for the systems with the most accurate constraints on system parameters such as masses, inclinations and orbital periods. We find that Gaia provides accurate distances in particular for systems which are at most a few hundred parsec away. This allows us to predict the gravitational wave amplitude $(\mathcal{A})$ with an accuracy better than $5 \%$ in the case of CD$30^{\circ} 11223$ and around $10 \%$ for AM CVn itself, making these systems ideal for the performance validation of LISA. For the remaining systems with distances of a few hundred parsec (e.g. HP Lib and SDSS J0923), the uncertainty of the gravitational wave amplitude is now dominated by the uncertainty on the component masses. For these systems and future discoveries precise mass measurements are required to provide estimations on the gravitational wave strength with a precision of a few percent.

\section{ACKNOWLEDGMENTS}

TK would like to thank Thomas Tauris for useful comments on the manuscript. VK would like to thank Tommaso Marchetti for useful discussion on derivation of Gaia distances. This work presents results from the European Space Agency (ESA) space mission Gaia. Gaia data is being processed by the Gaia Data Processing and Analysis Consortium (DPAC). Funding for the DPAC is provided by national institutions, in particular the institutions participating in the Gaia MultiLateral Agreement (MLA). The Gaia mission website is https://www.cosmos.esa.int/gaia. The Gaia archive website is https://archives.esac.esa.int/gaia. Armagh Observatory and Planetarium is core funded by the Northern Ireland Executive through the Dept. for Communities. This research made use of NumPy (Van Der Walt et al. 2011) This research made use of matplotlib, a Python library for publication quality graphics (Hunter 2007) This research made use of Astropy, a community-developed core Python package for Astronomy (Astropy Collaboration et al. 2013)

\section{REFERENCES}

Althaus L. G., Miller Bertolami M. M., Córsico A. H., 2013, A\&A, 557, A19

Amaro-Seoane P., et al., 2017, preprint, (arXiv:1702.00786)

Astraatmadja T. L., Bailer-Jones C. A. L., 2016, ApJ, 832, 137

Astropy Collaboration et al., 2013, A\&A, 558, A33

Bailer-Jones C. A. L., 2015, PASP, 127, 994

Bailer-Jones C. A. L., Rybizki J., Fouesneau M., Mantelet G., Andrae R., 2018, preprint, (arXiv: 1804.10121)

Bellm E., 2014, in Wozniak P. R., Graham M. J., Mahabal A. A., Seaman R., eds, The Third Hot-wiring the Transient Universe Workshop. pp 27-33 (arXiv: 1410.8185)

Bloemen S., Groot P., Nelemans G., Klein-Wolt M., 2015, in Rucinski S. M., Torres G., Zejda M., eds, Astronomical Society of the Pacific Conference Series Vol. 496, Living Together: Planets, Host Stars and Binaries. p. 254

Breivik K., Kremer K., Bueno M., Larson S. L., Coughlin S., Kalogera V., 2018, ApJ, 854, L1

Brown W. R., Kilic M., Allende Prieto C., Kenyon S. J., 2010, ApJ, 723, 1072

Brown W. R., Kilic M., Hermes J. J., Allende Prieto C., Kenyon S. J., Winget D. E., 2011, ApJ, 737, L23

Brown W. R., Gianninas A., Kilic M., Kenyon S. J., Allende Prieto C., 2016a, VizieR Online Data Catalog, 181

Brown W. R., Gianninas A., Kilic M., Kenyon S. J., Allende Prieto C., 2016b, ApJ, 818, 155

Brown W. R., Kilic M., Kenyon S. J., Gianninas A., 2016c, ApJ, 824,46

Carter P. J., et al., 2013, MNRAS, 429, 2143

Copperwheat C. M., Marsh T. R., Dhillon V. S., Littlefair S. P., Hickman R., Gänsicke B. T., Southworth J., 2010, MNRAS, 402, 1824

Cutler C., 1998, Phys. Rev. D, 57, 7089

Debes J. H., Kilic M., Tremblay P.-E., López-Morales M., Anglada-Escude G., Napiwotzki R., Osip D., Weinberger A., 2015, AJ, 149, 176

Espaillat C., Patterson J., Warner B., Woudt P., 2005, PASP, 117,189

Fontaine G., et al., 2011, ApJ, 726, 92

Gaia Collaboration et al., 2016, A\&A, 595, A1 
Gaia Collaboration Brown A. G. A., Vallenari A., Prusti T., de Bruijne J. H. J., Babusiaux C., Bailer-Jones C. A. L., 2018, preprint, (arXiv:1804.09365)

Geier S., et al., 2013, A\&A, 554, A54

Green M. J., et al., 2018a, preprint, (arXiv:1804.07138)

Green M. J., et al., 2018b, MNRAS, 476, 1663

Hermes J. J., et al., 2012, ApJL, 757, L21

Hunter J. D., 2007, Computing In Science \& Engineering, 9, 90

Igoshev A., Verbunt F., Cator E., 2016, A\&A, 591, A123

Israel G. L., Panzera M. R., Campana S., Lazzati D., Covino S., Tagliaferri G., Stella L., 1999, A\&A, 349, L1

Israel G. L., et al., 2002, A\&A, 386, L13

Istrate A. G., Tauris T. M., Langer N., Antoniadis J., 2014, A\&A, 571, L3

Kilic M., Brown W. R., Hermes J. J., Allende Prieto C., Kenyon S. J., Winget D. E., Winget K. I., 2011, MNRAS, 418, L157

Kilic M., Brown W. R., Gianninas A., Hermes J. J., Allende Prieto C., Kenyon S. J., 2014, MNRAS, 444, L1

Knigge C., 2006, MNRAS, 373, 484

Korol V., Rossi E. M., Groot P. J., Nelemans G., Toonen S., Brown A. G. A., 2017, MNRAS, 470, 1894

Kremer K., Breivik K., Larson S. L., Kalogera V., 2017, ApJ, 846, 95

Kupfer T., et al., 2015, MNRAS, 453, 483

Levitan D., et al., 2014, ApJ, 785, 114

Lindegren L., et al., 2018, preprint, (arXiv:1804.09366)

Littenberg T. B., Larson S. L., Nelemans G., Cornish N. J., 2013, MNRAS, 429, 2361

Liu J., Zhang Y., Han Z., Zhang F., 2010, Ap\&SS, 329, 297

Luri X., et al., 2018, preprint, (arXiv: 1804.09376)

Lyne A. G., et al., 2004, Science, 303, 1153

Macfarlane S. A., Toma R., Ramsay G., Groot P. J., Woudt P. A., Drew J. E., Barentsen G., Eislöffel J., 2015, MNRAS, 454, 507

Marchetti T., Rossi E. M., Brown A. G. A., 2018, preprint, (arXiv: 1804.10607)

Moore C. J., Cole R. H., Berry C. P. L., 2015, Classical and Quantum Gravity, 32, 015014

Motch C., Haberl F., Guillout P., Pakull M., Reinsch K., Krautter J., 1996, A\&A, 307, 459

Nelemans G., 2013, in Auger G., Binétruy P., Plagnol E., eds, Astronomical Society of the Pacific Conference Series Vol. 467, 9th LISA Symposium. p. 27 (arXiv:1302.0138)

Nelemans G., Jonker P. G., 2010, New Astron. Rev., 54, 87

Nelemans G., Steeghs D., Groot P. J., 2001a, MNRAS, 326, 621

Nelemans G., Yungelson L. R., Portegies Zwart S. F., 2001b, A\&A, 375, 890

Nelemans G., Yungelson L. R., Portegies Zwart S. F., 2004, MNRAS, 349, 181

Nissanke S., Vallisneri M., Nelemans G., Prince T. A., 2012, ApJ, 758,131

Patterson J., et al., 2002, PASP, 114, 65

Patterson J., et al., 2005, Publications of the Astronomical Society of the Pacific, 117, 1204

Provencal J. L., et al., 1997, ApJ, 480, 383

Ramsay G., Cropper M., Wu K., Mason K. O., Hakala P., 2000, MNRAS, 311, 75

Ramsay G., Hakala P., Cropper M., 2002, MNRAS, 332, L7

Ramsay G., Hakala P., Wu K., Cropper M., Mason K. O., Córdova F. A., Priedhorsky W., 2005, MNRAS, 357, 49

Roelofs G. H. A., Groot P. J., Marsh T. R., Steeghs D., Nelemans G., 2006, MNRAS, 365, 1109

Roelofs G. H. A., Groot P. J., Nelemans G., Marsh T. R., Steeghs D., 2007a, MNRAS, 379, 176

Roelofs G. H. A., Nelemans G., Groot P. J., 2007b, MNRAS, 382, 685

Roelofs G. H. A., Groot P. J., Benedict G. F., McArthur B. E., Steeghs D., Morales-Rueda L., Marsh T. R., Nelemans G., 2007c, ApJ, 666, 1174
Roelofs G. H. A., Rau A., Marsh T. R., Steeghs D., Groot P. J., Nelemans G., 2010, ApJL, 711, L138

Ruiter A. J., Belczynski K., Benacquista M., Holley-Bockelmann K., 2009, ApJ, 693, 383

Ruiter A. J., Belczynski K., Benacquista M., Larson S. L., Williams G., 2010, ApJ, 717, 1006

Shah S., Nelemans G., 2014, ApJ, 790, 161

Shah S., van der Sluys M., Nelemans G., 2012, A\&A, 544, A153

Skillman D. R., Patterson J., Kemp J., Harvey D. A., Fried R. E., Retter A., Lipkin Y., Vanmunster T., 1999, PASP, 111, 1281

Solheim J. E., 2010, Publications of the Astronomical Society of the Pacific, 122, 1133

Steeghs D., 2017, Nature Astronomy, 1, 741

Steeghs D., Marsh T. R., Barros S. C. C., Nelemans G., Groot P. J., Roelofs G. H. A., Ramsay G., Cropper M., 2006, ApJ, 649,382

Ströer A., Vecchio A., 2006, Classical and Quantum Gravity, 23, 809

Strohmayer T. E., 2004, ApJL, 608, L53

Strohmayer T. E., 2005, ApJ, 627, 920

Thorstensen J. R., 2003, AJ, 126, 3017

Thorstensen J. R., Lépine S., Shara M., 2008, AJ, 136, 2107

Toonen S., Hollands M., Gänsicke B. T., Boekholt T., 2017, A\&A, 602, A16

Vallisneri M., 2005, Phys. Rev. D, 72, 042003

Van Der Walt S., Colbert S. C., Varoquaux G., 2011, Computing in Science \& Engineering, 13, 22

Wevers T., et al., 2016, MNRAS, 462, L106

Yu S., Jeffery C. S., 2010, A\&A, 521, A85 